\title{
Il feto e la ricerca del sé. Un dialogo critico con Merleau-Ponty
}

Fabio Di Clemente*

Riassunto: Alla luce della vita prenatale, radicalizziamo la riabilitazione del corpo sostenuta da Merleau- Ponty. Attribuiamo al feto la partecipazione al fenomeno del "self". In particolare, riabilitiamo il feto mediante l'analisi della processualità che lo costituisce senza un principio previamente dato. Lo scopo principale è quello di pensare la comparsa del feto criticando i privilegi che Merleau-Ponty indica per il mondo postnatale.

Parole chiave: Feto. Sé. Corpo. Carne. Merleau-Ponty.

\section{The fetus and the search for the Self. A critical dialogue with Merleau-Ponty}

\begin{abstract}
In the light of prenatal life, we radicalise the rehabilitation of the body argued by Merleau-Ponty. We attribute to the fetus the participation in the phenomenon of the "self". In particular, we rehabilitate the fetus through the analysis of the processuality which constitutes it without a principle previously given. The principal purpose is to think about the appearance of the fetus criticizing the privileges that Merleau-Ponty points to in the postnatal world.
\end{abstract}

Keywords: Fetus. Self. Body. Flesh. Merleau-Ponty.

\section{O feto e a procura do Si. Um diálogo crítico com Merleau-Ponty}

Resumo: À luz da vida pré-natal, radicalizamos a reabilitação do corpo sustenta-

* Doutor em Filosofia pela Universidade dos Estudos de Urbino Carlo Bo (Itália). Prof. Adjunto de Filosofia na Universidade Federal do Espírito Santo. E-mail: fdicle@libero.it 
da por Merleau-Ponty. Atribuimos ao feto a participação ao fenômeno do "self". Em particular, reabilitamos o feto mediante a análise da processualidade que o constitui sem um princípio previamente dado. Nosso intuito principal é pensar o aparecimento do feto criticando os privilégios que Merleau-Ponty aponta para o mundo pós-natal.

Palavras-chave: Feto. Si. Corpo. Carne. Merleau-Ponty.

\section{Il feto tra il sistema di risposta, il Sé e l'altro da Sé}

Nello sviluppo del feto ci imbattiamo nella relazione tra comportamento e maturazione cerebrale su un più ampio sfondo vitale, esibito dalla processualità maturativa.

Contestualmente al sistema di risposta, le ricerche pionieristiche di Milani Comparetti (1981) parlavano di un «repertorio dei pattern motori primari, moduli di movimento che si vedono comparire tra la $10^{\mathrm{a}}$ e la $20^{\mathrm{a}}$ settimana di età gestazionale» (Righetti 2003: 56). Secondo la «mappa del vissuto» di Ianniruberto e Tajani (1981), a sole otto settimane, ai movimenti vermicolari, ai «movimenti lievi e frequenti che interessano l'intero embrione», si alternano «movimenti globali del corpo (startle)» (Manfredi e Imbasciati 2012: 42). ${ }^{1}$ A quattordici settimane i movimenti sono sempre più ampi ed apparirebbe il fenomeno dell'abituazione, da cui dedurre «una qualche forma di memoria nel feto» e «l'esistenza e la funzionalità di una struttura mentale, quale che sia» (ivi: 42). Nella $16^{\text {a }}$ settimana emergono significativamente i comportamenti esplorativi, con un buon coordinamento temporo-spaziale: le mani toccano le pareti

1 Negli anni ottanta, vari autori, tra cui Vries, Visser e Prechtl, hanno attribuito l'esistenza della motricità allo stesso periodo (cfr. De Vries, Visser e Prechtl 1985, cit. in Manfredi e Imbasciati 2012: 43). In particolare, come riferiscono Manfredi e Imbasciati su queste ricerche, il repertorio dei movimenti, che all'inizio presenta una lenta flessione ed estensione della colonna vertebrale con passivo spostamento degli arti superiori e inferiori, si espande alla decima settimana. 
uterine placentari, afferrano il cordone ombelicale e si afferrano anche l'una con l'altra; inoltre, esse toccano la testa, il tronco e i piedi (cfr. ivi: $42,43)$. Sempre secondo la mappa di Inniruberto e Tajani, intorno alla $20^{\mathrm{a}}$ settimana di gestazione il feto sviluppa la volontarietà dei movimenti, indicata da un'attività esplorativa rivolta «non più solo all'ambiente, ma anche direttamente a se stesso, possibile grazie alla coordinazione motoria maturata dei movimenti degli arti» (ivi: 43). Altra fase rilevante avviene dalla $27^{\mathrm{a}}$ settimana, quando il feto produce il comportamento in senso stretto, dato dal momento in cui esso è «in grado di percepire uno stimolo sonoro esterno e di differenziare il suo comportamento al variare dello stimolo»: c'è una differenziazione della motricità che si fa comportamento, una volta tolta la funzione motoria - data dai tre tipi di repertori («"genetico"», «“epigenetico"» e «“appreso"») - dalla stretta dipendenza dagli stimoli (Righetti 2003: 56-57).

Riguardo alla relazione tra le parti e il tutto e alla critica delle garanzie metafisiche classiche, va evidenziato che sono presenti negli embrioni animali «movimenti a cadenza ritmica a cui tutte le parti del corpo in grado di muoversi parteciperebbero, comportamenti non coordinati (in numerose specie) [...]». Di tali movimenti è escluso significativamente ogni principio previamente dato, davanti alla difficoltà di comprenderne «la ragione d'esistenza»: risulterebbe inapplicabile tanto «l'interpretazione di un'utilità» per spiegare i comportamenti adulti, quanto «la finalità contingente, movimenti cioè attivati in risposta a stimoli sensoriali, nell'ottica per cui il feto sarebbe in grado di muoversi per garantirsi un minimo di benessere o per sentirsi protetto» (Manfredi e Imbasciati 2012: 41). Sembra imporsi una vera e propria iniziazione di tipo orgânico-comportamentale, su uno sfondo comportamentale confuso: davanti all'ordine che interessa anche le sequenze di movimenti non cooordinati, le «prime attivazioni di muscoli specifici, gli estensori ed i flessori che presiedono la deambulazione precoce», nel periodo fetale ad essere messe a punto sono inizialmente «circoscritte aree motorie di elevata competenza e maturità in un insieme comportamentale che appare 
invece confuso, mentre l'integrazione di specifiche competenze motorie in uno schema organizzato, complesso e significativo sarebbe rimandata a successive fasi prenatali» (ivi: 41).

Sul piano filosofico, possiamo dire correlativamente che, preso nel complesso della sua attività comportamentale, il feto esibisce l'originario corpo vissuto esistenzialmente, piuttosto che come oggetto intenzionale, conosciuto. È il feto a far uso del suo corpo stando nella situazione del «corpo eclissato» postnatale, di cui ci parla Merleau-Ponty, ossia stando all'interno di una «mediazione corporea che gli sfugge» (FP: 206). ${ }^{2}$ Le performance del feto indicano che, dalla condizione eminente di corpo eclissato, esso conquista un margine di volontarietà, di scelta, di desiderio, togliendo i movimenti da un'ipotetica processualità puramente meccanica o completamente involontaria. Quanto dicono Gallagher e Zahavi (2009: 224) per le nostre azioni più abituali (l'andatura, l'afferrare un oggetto ecc.) del mondo postnatale, dialogando con l'eredità della fenomenologia, possiamo dire del feto quando esegue movimenti: il feto può agire uscendo dall'ordine riflessogeno senza far uso di alcuna consapevolezza esplicita, oltre che - come vedremo - di una "memoria esplicita". Piuttosto, considerate le performance del feto, andrebbe capito nella fase prenatale ciò che è affermato in ambito postnatale: ci riferiamo alla portata critica del fatto che anche quando il bambino o l'adulto eseguono movimenti, in cui si ipotizza che certi dettagli dei processi di controllo o gli stessi processi rimangano inconsci, i movimenti stessi non sono inconsci, involontari o meccanici, ma fanno parte di un' «intenzionalità operativa», così come «sono sentiti in maniera immediata e preriflessiva» (ivi: 224. Cfr. FP: 200).

2 Nella prima opera, Merleau-Ponty scrive al riguardo: «La mediazione corporea mi sfugge quasi sempre; quando assisto ad eventi che mi interessano non ho coscienza delle cesure continue che il battito delle palpebre impone allo spettacolo e queste cesure non figurano nel mio ricordo [...]. In realtà, il corpo proprio e i suoi organi rimangono i punti di appoggio o i veicoli delle mie intenzioni, e non sono ancora colti come "realtà fisiologiche") (SC: 206). 
Il livello comportamentale conduce a dar conto del secondo siste$\mathrm{ma}$, rappresentato dalla ricezione. In ambito psicofisiologico emerge la capacità complessiva del feto di interagire con l'utero: prima dell'affermarsi del livello di elaborazione dei processi cerebrali e della loro influenza sull'amigdala (livello individuato durante la $30^{\mathrm{a}}$ settimana), notoriamente vi è già la partecipazione della sensorialità allo sviluppo del feto. In effetti, vi è una precoce complessità fetale esibita a partire dalla $20^{\mathrm{a}}$ settimana di gestazione: il feto è sensibile ad una complessità di stimoli, da quelli tattili, cinestesici, uditivi, gustativi e luminosi a quelli termici e dolorifici (cfr. Koupernick e Arfouilloux 1970, cit. in Mancia 2006: 209). ${ }^{3}$ Le ricerche pionieristiche di Milani Comparetti (1981) ci dicevano che dopo la $20^{\mathrm{a}}$ settimana di gestazione «il feto risponde attivando e coordinando più parti del suo corpo» (Righetti 2003: 55): il tempo di reazione tra l'emissione dello stimolo, la ricezione e la risposta ad esso è più lungo, il che fa avanzare l'ipotesi di un'elaborazione dello stimolo in entrata secondo una decisione volontaria (cfr. $i b i d$.). Si tratta, dal punto di vista neurofisiologico, di una vera e propria «interazione sensomotoria materno-fetale», secondo costanza e ritmicità caratterizzanti le funzioni di contenimento del feto (Mancia 2006: 209). Relativamente ai sensi, sul piano scientifico si parla della presenza di «una continuità tra la fase prenatale e la fase postnatale, nonostante sia differente l'ambiente in cui vive il feto» (BUSNEL 2008: 15). Significativamente, i dati ci parlano di una sensorialità completa del feto; sensorialità che la scienza anticamente negava perché - dice la Busnel - il sistema nervoso non è anatomicamente completo. Siamo di fronte ad una sensorialità che ci restituisce il feto nella sua integrità e che è valutata come comprovazione dell'esistenza di un «essere umano», anche se la dimostrazione scientifica dell'esercizio del pensiero da parte del feto rimarrebbe un problema (ivi: 15).

\footnotetext{
3 Per una visione complessiva sulla sensorialità del feto, rimandiamo a Herbinet e Busnel (2001).
} 
Se i dati riportati precedentemente aiutano a pensare alla complessività delle capacità fetali, l'uso che di esse se ne fa finisce per privilegiare un sistema rispetto all'altro, una competenza rispetto all'altra, uno stato (motorio, sensoriale, emotivo, cognitivo, mnestico, onirico ecc.) rispetto all'altro. Di contro, alla luce dei problemi trascendentali che pone la processualità maturativa vitale, privilegiare un aspetto rispetto all'altro può comportare solo l'accentuazione della natura operativa di uno di essi per distinguerla dalle altre. Senza poter approcciare i privilegi per i vari stati e per le varie fonti di conoscenza, notiamo, ad esempio, che vi è l'enfasi posta sul tatto, considerato come la prima sensorialità in via di sviluppo del feto (Montagu: 1978). In alternativa, non manca l'attribuzione di un peso fondamentale all'olfatto: esso diventa la capacità percettiva «fondamentale», in quanto "“filo conduttore chimico" che, attraverso la discontinuità della nascita, permette al neonato di orientarsi nell'ambiente postnatale» (Schaal e Hertling 2001: 362). In sede di psicologia clinica, autori menzionati precedentemente hanno dato un «ruolo centrale» all'esperienza auditiva rispetto alle prime elaborazioni esperienziali del feto, secondo una visione costruttivistica (cfr. Manfredi e Imbasciati 2012: 28). Tuttavia tali scelte non rendono conto della natura di un continuum non previamente dato, né realizzabile una volta per tutte, che la processualità maturativa vitale intrauterina ci restituisce. Come vedremo, la processualità maturativa vitale del prenatale agisce come condizione di possibilità di qualunque primato o privilegio che vi si voglia costruire sopra.

A questo punto, dobbiamo approcciare anche il terzo sistema: l'elaborazione cerebrale e psichica del feto. S'impone la distinzione tra il Sé, l'ambiente (vitale e di comportamento) e l'altro da Sé finanche nella vita intrauterina, che problematizza non solo i privilegi summenzionati (per non parlare dei tanti altri), ma anche quelli ontologici attribuiti da Merleau-Ponty al mondo postnatale.

Notoriamente, il riconoscimento della percezione che sia ad un tempo del sé e dell'altro è posticipato da Merleau-Ponty - sulla base 
degli studi di Wallon (1976: 239-260) - all'età di sei mesi. Prima di ques'età, oltre al problema della "nozione visiva del proprio corpo", su cui torneremo più avanti, vi sarebbe il problema della mancanza di saldatura tra l'esterocettivo e l'introcettivo e dell'immaturità dell'equilibrio corporeo (cfr. RAE: 188). Ma ciò che egli evidenzia con Wallon come «cose necessarie alla percezione» postnatale (la «coscienza globale della posizione del mio corpo nello spazio, i riflessi correttivi che sono necessari ad ogni istante e la coscienza della spazialità del mio corpo»), congiuntamente alla «saldatura tra i due domini, l'esterocettivo e l'introcettivo» (RAE: 183), finisce per risultare assolutamente difficile da separare all'età zero e finanche nel prenatale. Oggi sappiamo quanto sia molto più ricco osservare sin dall'età zero il nascituro. Ad esempio, volendo limitarci ad autori in dialogo con la fenomenologia, gli studi sull'imitazione, in cui si è evidenziata tanto la capacità di imitare l'altro quanto quella di correggere i propri movimenti, ci dicono che per i «neonati c'è una differenziazione rudimentale tra il sé e il non-sé», per cui non possiamo escludere dalle «prime esperienze» il «senso di sé e dell'altro». Ne segue la conclusione, contro Wallon e Merleau-Ponty: «La connessione sperimentale tra il sé e l'altro è operante a partire dalla nascita [...]» (Gallagher e Meltzoff 2010: 121).

Ma guardando al prenatale, ci imbattiamo in una radicalizzazione della ricerca del senso corporeo e del self. Ad apparire è il «Proto-Self», il «Self neurobiologico», quale originaria espressione della mente del feto. Il Self neurobiologico funziona nel feto come «un modulatore della omeostasi», dell'equilibrio che sostenta la vita, capace di creare per il feto «una coscienza dei propri limiti somatici che gli permetta di distinguere alla nascita il suo Sé dall'altro Sé» (Mancia 2006: 212). La coscienza del feto è vista come qualcosa che «potrebbe essere in grado di creare proto-rappresentazioni» (ivi: 210). Al di là del ricorso al concetto di rappresentazione, che sarà oggetto di critica dal nostro punto di osservazione, è rilevante sostenere che la coscienza dei limiti somatici, foriera della propriocezione, vada attribuita originariamente 
al feto. Incisivamente, nel campo della neuroscienza, in una prospettiva che vede il corpo essere il fondamento della mente, viene radicalizzata ulteriormente la nozione di protoself, congiuntamente a quella di vita. Vi sarebbe un sentimento elementare dell'esistenza che non rimonta semplicemente alla corteccia cerebrale, ma al self primordiale, a ciò che recupera finanche la componente del tronco cerebrale per il sorgimento della mente. I sentimenti primordiali diventano i costituenti della mente, del self, prima che essi siano attribuibili esclusivamente alla corteccia cerebrale (cfr. Damasio 2011: 102). In maniera ancora più radicale, questo «sentimento primitivo/self primordiale» sarebbe un «prodotto spontaneo del protoself», nel senso che i sentimenti primordiali occorrerebbero indipendentemente dal fatto che il protoself stia o no "occupato con oggetti ed eventi esterni al cervello». Piuttosto, i sentimenti necessitano di «stare relazionati al corpo vivo, e nulla più». Dunque, nella scala di costruzione del self, vi sarebbe originariamente finanche un protoself che non è ancora il «self centrale», ciò che «include un sentimento di conoscere in relazione ad un oggetto» (Damasio 2011: 389-390, n. 17). ${ }^{4}$

Alcuni dati sperimentali ci dicono che è possibile pensare la propriocezione come base non cosciente nella formazione dello schema corporeo. Essa può essere compresa secondo una doppia funzione: per un lato, la propriocezione «si compone di una informazione fisiologica non cosciente» che dà al corpo il senso della sua postura e del suo movimento,

4 Senza poter discutere in questa sede i presupposti dell'opera di Damasio, va rilevato che, non differentemente da Mancia, si ricorre ad un problematico ed equivoco concetto di rappresentazione. Si afferma che il protoself diventa una «collezione integrata di modelli neuronali separati che mappano, in ogni momento, gli aspetti più stabili della struttura fisica dell'organismo». Certamente, sono mappe del protoself che «generano non solo immagini corporali, ma anche immagini corporali sentite» (Damasio 2011: 235-236). Nondimeno, il protoself è ciò che produce un insieme di sentimenti connessi ad una "rappresentazione integrata" dell'organismo; rappresentazione che condurrebbe gradualmente alla coscienza. Nella produzione della «mente cosciente» vi sarebbe una «serie di immagini», ovvero, una «immagine dell'organismo [...] data dalla rappresentazione del protoself modificato» (ivi: 251), così come l' «organismo» è «rappresentato», in uno stadio anteriore, dallo stesso protoself (ivi: 225). 
quindi «gioca un ruolo essenziale nel sistema dello schema corporeo»; per l'altro, questa stessa informazione propriocettiva può essere «la base di una coscienza corporea», in ragione della quale si può dire dove sono le proprie gambe anche se si hanno gli occhi chiusi. È una tale coscienza propriocettiva, incorporata all'informazione fisiologica non cosciente, ad aiutarci a costituire infine l'aspetto percettivo dell'immagine del corpo. In particolare, questa doppia funzione della propriocezione aiuterebbe a spiegare anche «la comunicazione intermodale tra la visione e la propriocezione»; interconnessione che è allo stesso tempo «una comunicazione tra gli aspetti sensoriali e motori del comportamento». Di qui, diventano significativi gli studi sull'imitazione infantile che suggeriscono che il nascituro possiede sin dall' anno zero tanto uno «schema corporeo primitivo (un sistema che funziona automaticamente per rendere possibile la coordinazione delle posture e del movimento) quanto una immagine del corpo primitivo - una coscienza propriocettiva, i.e. del suo viso [...]» (Gallagher e Meltzoff 2010: 108). ${ }^{5}$ Ora, la propriocezione è attribuibile

5 Bisogna notare che Gallagher è stato giustamente criticato da Saint Aubert. La differenza che passa tra l'immagine del corpo e lo schema del corpo sarebbe simile a quella che passa «tra una percezione (analisi o controllo) del movimento e l'accompagnamento attuale del movimento» (Gallagher e Meltzoff 2010: 91; cfr. Gallagher e Zahavi 2009: 225). Una tale distinzione tra schema corporeo e immagine corporea non si allinea con l'impostazione di Merleau-Ponty, per il quale l'immagine corporea non è concepita in chiave di coscienza percettiva deputata all'analisi e al controllo. Inoltre, la conseguente distinzione concettuale tra motricità e percezione che ne consegue non ci restituirebbe sul piano empirico e filosofico lo schema corporeo cercato da Merleau-Ponty. Nella misura in cui - nota Saint Aubert contro Gallagher - la coscienza percettiva per Merleau-Ponty diventa espressione di un «"modo d'accesso all'essere"», e non di un «soggetto regionale», in essa confluiscono le varie «modalità» («intersensoriali e intermodali, immaginarie ed espressive»), fino ad incorporare quelle relative al «desiderio» e alla «profondità» (Saint Aubert 2013: 57; cfr. MSME: 46). Infine, sugli altri limiti di natura epistemologica interni all'impiano che nutre tale lettura (l'acquisito e l'innato, il personale e il pre-personale, il volontario e l'automatico, il cosciente e l'incosciente) ci limitiamo a rimandare sempre a Saint Aubert (ivi: 39-59). Al contempo, fatte salve queste critiche, riteniamo che, 
allo stesso feto (Gallagher e Zahavi 2009: 220), ad un feto che dagli studi più recenti sorprende per il livello delle sue performance che va ben oltre le funzioni espresse dalla stessa propriocezione.

Vi è una complessa maturazione del feto, di cui parla la neurofisiologia in dialogo con la psicoanalisi, in cui non è possibile tracciare una frontiera precisa tra il fisiologico e lo psicologico. Si tratta di «una processualità maturativa affidata a "organizzatori” precoci di funzioni motorie, respiratorie, vegetative, sensoriali e integrative correlate con cicli di riposo e attività del feto quali precursori del sonno quieto e attivo, quest'ultimo precursore a sua volta del sonno Rem del neonato». $\mathrm{Ci}$ troviamo di fronte a funzioni che formerebbero «un apparato con cui il feto si mette in relazione con la madre e l'ambiente uterino che lo contiene», di funzioni fortemente rilevanti anche per la psicoanalisi, nella misura in cui essa studia «le più precoci relazioni d'oggetto, le loro dinamiche e le conseguenze emozionali e cognitive che queste producono» (Mancia 2006: 207). Significativamente, nelle fasi più tardive di gestazione, abbiamo le funzioni integrative del feto. Sulla base di altri studi (Joseph 2000), sappiamo che esse «compaiono in forma completa

nonostante il fatto che nel pensiero di Merleau-Ponty l'immagine del corpo sia profondamente relazionata allo schema corporeo e non sia conseguentemente quella proposta da Gallagher, la distinzione operata da quest'ultimo conservi un suo valore teoretico nella misura in cui, oltre ad evitare la confusione tra i due concetti sul piano scientifico, mette in luce l'istanza critica interna alla nozione di schema corporeo, che è in parte anche quella di Merleau-Ponty: dover rimanere all'altezza dello schema corporeo significa rimanere all'altezza primieramente di un'iniziazione comportamentale al mondo senza ricorrere alla "rappresentazione" (Vorstellung) e ad un'immagine speculare, dunque di rimanere all'altezza di ciò che «implica certe capacità motrici, abilità e abitudini che rendono possibile il movimento e il mantenimento della postura» (Gallagher e Meltzoff 2010:91) senza ricorrere-aggiungiamo - all'unità fenomenica e ontologica prodotta da un'ipotetica immagine presa nello specchio-strumento o solamente nel fantasma. Come vedremo, sarà questo superamento dell'immagine nello specchio-oggetto o dell'immagine-fantasma ad essere perseguito da Merleau-Ponty, ma non prima di aver aderito per lo meno all'idea dell'immagine presa ad un fantasma. 
quando la maturazione del tronco encefalico è molto avanzata, circa alla $28^{\mathrm{a}}$ settimana, e quando la corteccia cerebrale è sufficientemente formata da potersi integrare con le strutture sottocorticali e permettere così l'elaborazione di un contenuto sensoriale» (Mancia 2006: 209). Alla formazione della corteccia cerebrale, circa alla $36^{\mathrm{a}}-38^{\mathrm{a}}$ settimana gestazionale, resterebbe così vincolata l'elaborazione di un contenuto sensoriale. È «il momento in cui la corteccia cerebrale maturata anche grazie agli impulsi sinaptogenici provenienti dal tronco, diventa il luogo in cui le esperienze sensoriali (ad esempio acustiche e somoestesiche) possono permettere al feto una forma di coscienza a contenuto sensoriale». Sulla base degli studi di Burgess e Tawia (1996), il periodo di fine gestazione diventa la fase in cui si forma la coscienza come «vigilanza» (Mancia 2006: 210). S'impone una distinzione di qualche natura tra il Sé, l'ambiente e l'altro da Sé.

Nella sua specificità fisiologica e nel suo ambiente originale e originario, il feto realizza movimenti generali (fino a tredici settimane) che «non sono stereotipati. Mentre li esegue, esso si adatta costantemente alle condizioni ambientali interne ed esterne» (Piontelli 2007: 426). Nonostante le varie cautele, risulta difficile negare al feto maturo «il senso corporeo», presente «forse già in un'epoca molto precoce della gestazione», che «permetterebbe all'organismo di adattarsi al continuo mutare delle condizioni interne e ambientali». Richiamando il Self di Damasio, si afferma che durante le «fasi successive della gestazione il senso corporeo potrebbe evolvere in un "Sé nucleare" o in un "Sé percettivo e motorio" embrionali» (ivi: 418). ${ }^{6}$ Di qui, in luogo di negare al feto la percezione, si tratta di distinguere tra «feto attivo dei primi tre mesi e feto maturo dell'imminenza della nascita», riconoscendo al primo

6 Toccare il cordone ombelicale e le parti dell'ambiente uterino, diventa l'occasione per «[...] una quantità di contatti con elementi estrinseci e abbondanti feedback sensomotori», così come il fatto di toccare regolarmente il proprio corpo rende possibile l'emergenza di un «[...] qualche senso corporeo o protoSé» (Piontelli 2007: 417) 
il «sentire», «il ricevere informazioni sull'ambiente esterno», mentre al secondo il «percepire», «l'interpretazione delle sensazioni», che comporta attribuire loro un significato (Piontelli 2007: 417). Relativamente al sonno, la studiosa aggiunge: «Diversamente da ciò che avviene nella vita postnatale, il feto probabilmente è in grado di "conoscere" e dominare il proprio ambiente durante il sonno attivo» (ivi: 431).

La percezione di sé e del proprio ambiente viene estesa all'altro feto. In caso di parto gemellare, l'esplorazione è capace di rivolgersi all'altro, secondo caratteristiche che si ritrovano finanche nella relazione di tipo sociale. Al riguardo, particolarmente significative sono le rigorose ricerche sulla natura cinematica dei movimenti di feti singoli e dei movimenti in gravidanze gemellari. Con gli studi sui feti singoli (cfr. Zoia et al., 2007), dalla $22^{\mathrm{a}}$ settimana di gestazione sono stati individuati movimenti coordinati, caratterizzati da una pianificazione in relazione allo spazio e al tempo e da una modulazione sull'obiettivo (occhi, bocca, parete uterina). Successivamente, l'estensione di questi studi cinematici alle coppie di feti, con l'uso dell'ecografia quadrimensionale (4D - US), ha fatto emergere una differenziazione della motricità sul piano intercorporeo: alla pianificazione motoria, si aggiungono le differenze cinematiche nel movimento, a seconda che il movimento sia diretto verso il proprio corpo, la parete uterina o l'altro gemello. Incisivamente, si è dimostrato che lo schema cinematico dei movimenti diretti verso il co-gemello è diverso da quello che interessa i movimenti rivolti verso sé e verso la parete uterina. Inoltre, si afferma un adattamento cinematico al target che diventa del tutto peculiare con il co-gemello. I movimenti diretti verso il proprio corpo diminuiscono tra la $14^{\mathrm{a}}$ e la $18^{\mathrm{a}}$ settimana gestazionale, mentre quelli diretti più lontano dal proprio corpo non mostrano differenze durante le varie fasi della gestazione. Ma cosa accade per i movimenti diretti verso il co-gemello? Essi aumentano progressivamente, fino a costituire $1129 \%$ dei movimenti osservati nella $18^{\mathrm{a}}$ settimana. Inoltre, vi è una differenza in merito alla durata e alla velocità dei movimenti, a seconda che siano rivolti verso sé, l'ambiente uterino e il co-gemello: quelli diretti verso 
quest'ultimo hanno una durata e una decelerazione maggiori rispetto agli altri due. Le decelerazioni si modellano poi in relazione al target che accompagna il gesto (parti maggiori o minori del corpo gemello, ginocchio o punta del naso, o parti più lontane dell'ambiente uterino). Tali differenze, omogenee nelle coppie di gemelli esaminati, ci dicono che il movimento verso il co-gemello è coordinato, pianificato e intenzionale, dunque socialmente orientato (cfr. Castiello et al. 2010). Come vedremo più avanti, una tale precocità del sistema motorio e di controllo, unitamente alla "percezione di" e finanche alla "co-percezione di" (di Sé, dell'ambiente uterino e dell'altro da Sé), ripropone evidentemente anche il problema dell'ontogenesi del sistema nervoso centrale.

Anticipiamo che non si tratta, scientificamente, di omologare il prenatale al postnatale né, filosoficamente, di ricucire un continuum con caratteristiche riduzionistiche o pregno di garanzie metafisiche (ad esempio, di quelle classiche dell'essenzialismo e del finalismo). Se, per un lato, non possiamo pensare ad un continuum che faccia dei fenomeni evolutivi «un processo continuo in cui gli eventi precoci sono collegati agli eventi posteriori da una successione di relazioni di causa ed effetto» (Piontelli 2007: 417), per l'altro, non possiamo «incorrere nell'errore di sostenere che il feto (o anche il neonato) non abbia alcun senso dei confini con il proprio ambiente. È stata postulata l'esistenza di una fase "fusionale"» (ivi: 426). Da un lato, non possiamo attribuire al comportamento del feto un significato che coincida con quello delle manifestazioni del neonato e addirittura dell'adulto (cfr. ibid.); dall'altro, aggiungiamo, sembra essere errato ridurre la vita fetale ad una serie di deficit rispetto al postnatale o, nel caso migliore, ad un fenomeno di sola "anticipazione" del postnatale. Al fine di evitare questo doppio errore, riteniamo che si debba riabilitare il prenatale come la "fondazione originaria" (Urstiftung) della nostra apertura al mondo e all' altro, della nostra frequentazione originale e originaria dell'Essere, differentemente anche dalla posizione di Merleau-Ponty ancorata agli studi dell'epoca. Ma ciò non significa che una tale riabilitazione debba avvenire contro l'impianto 
filosofico di quest'ultimo. In effetti, l'interrogazione merleau-pontiana ci permette di capire coerentemente il prenatale alla luce di una relazione carnale e d'essere.

\section{La ricerca del Sé carnale}

La rilevanza dei dati e fatti empirici fin qui esposti sul prenatale (così come di tanti altri che non possiamo qui riportare) suggeriscono che le reversibilità vitali e conoscitive debbano spostare il trascendentale verso il lato dell'ontogenesi intraorganico-comportamentale-ambientale del sé, in cui ritrovare emblematicamente, nel quadro critico merleau-pontiano, l'intreccio di anatomia e funzione, maturazione organica e apprendimento, motricità e percezione, postura e comportamento etc. Al contempo, il senso fisiologico originale e originario in Merleau-Ponty non può essere ridotto al sorgimento di una coscienza scientifica a contenuto sensoriale, così come la coscienza non può coincidere con l'aspetto della "vigilanza". ${ }^{7}$ La coscienza percettiva si lega tanto al fenomeno dell'espressione quanto alla relazione d'essere. Al contempo, ciò avviene a condizione che passi per l'eminenza della visione. Si tratta di un'eminenza che è rappresentata dalle dinamiche interne più varie del voyant- $v u$, che sono di natura neurologica, psicologica, psicoanalitica, non solo fenomenologica.

Come si è documentato in maniera consistente, nel prolungamento dello studio dello specchio di Wallon e Lacan, e sotto la decisiva influenza di Schilder, tutti gli ultimi scritti di Merleau-Ponty modellano la tematica del senziente-sentito sulla «figura capitale del voyant-vu»; è una figura che, nella misura in cui è «inizialmente più psicologica e psicanalitica che fenomenologica», induce a pensare che non siamo di

7 In uno spirito antidualista e antiriduzionista, attraverso il "problema della percezione", la filosofia merleau-pontiana mira a farsi «trascendentale, cioè radicale», non perché si insedia «nella coscienza assoluta senza menzionare $\mathrm{i}$ gradi cui è passata per giungervi, ma considerando se stessa come un problema $[\ldots] \gg($ FP: 108). 
fronte ad una «generalizzazione del tocante-toccato husserliano» (Saint Aubert 2013: 178-179). Il narcisismo del sensibile-senziente, così come quello del senziente-sentito, sono modellati su quello del voyant-vu. Di conseguenza, avere il «senso fisiologico» del corpo significa averlo già preso nelle «strutture percettive, espressive, desideranti». La giustificazione sta nel fatto che sono tali strutture ad essere deputate a trascinare con sé la trascendenza del nostro essere al mondo (cfr. ivi: 377). Solo in tal senso rimane la bilateralità tra schema corporeo e immagine corporea anche nell'interrogazione tardiva del filosofo francese. La reversibilità, posta nel fenomeno espressione, produce ultimamente un'esistenza dimensionale della percezione, in cui ritroveremo il rapporto ultimo più complesso, rispetto alla circolarità, all'Ineinander, all'empiétement: quello della profondità.

Tra "circolarità", "sopravanzamento" e "ricoprimento", Merleau-Ponty privilegia la visione come indice della trascendenza nell' ordine della segregazione, pensando secondo la modalità del "fantasma", di "qualcosa di noi stessi nelle cose", tra "introiezione" e "proiezione" (cfr. $\mathrm{N}$ : 399), "introiezione" ed "espulsione" (cfr. ivi: 398). La ragione sta nel fatto che, per un lato, lo specchio come strumento ci pone a distanza dal nostro corpo e, per l'altro, ci restituisce ciò che sta contenuto in essa, come accade quando nella nostra immagine dello specchio riotteniamo qualcosa di noi nelle cose che contempliamo ${ }^{8}$ : la visione ci conduce nel visibile che supera i limiti del corpo, ristabilendo, allo stesso tempo, l'unità nel mondo. Egli conclude affermando che la visione completa

8 Merleau-Ponty si rifà qui alle ricerche di Paul Schilder (1973: 262-263), autore che lo ha influenzato di forma decisiva sul tema, richiamando l'attenzione sul fenomeno della captazione: «Captazione del corpo tattile da parte dell'immagine visiva [...]: sento nello specchio il contatto della mia pipa con la mia mano» (N: 397). Si veda anche OS: 27. Sulla relazione tra carne e specchio, rimandiamo al prezioso contributo di Saint Aubert (2013: 165-200), in cui vi è la messa in luce della fonte decisiva e costante di Schilder sul tema specifico dello specchio, rispetto alle altre di Henry Wallon, Wolfgang Metzger, Jacques Lacan, Françoise Dolto e René Zazzo. 
il corpo estesiologico, mentre «la visualizzazione del tattile è un espediente» (ivi: 398$)^{9}$. In tal senso può scrivere che «[...] il nostro mondo è principalmente ed essenzialmente visivo; con dei profumi o dei suoni non formeremmo un mondo» (VI: 105).

Tuttavia, da un lato, dobbiamo dire che questa modalità dominante del campo percettivo stride primieramente con i dati scientifici, con le "provocazioni della scienza" a cui Merleau-Ponty stesso invitava a non sottrarsi; dall'altro, come ci accingiamo a notare, lo stesso privilegio della visione polarizzato sull'immagine-fantasma viene problematizzato e superato davanti ad altri aspetti della conoscenza legata ai virtuosismi della visione, indicati - anche se non sviluppati sistematicamente - dallo stesso filosofo francese: l'onirico, il desiderio e, infine, la profondità del mondo.

Riguardo alle "provocazioni delle scienze", nel fenomeno dell'imitazione del neonato vi è dall'età zero la manifestazione di una «nozione "primordiale" o "embrionale" del sé», di «un sé propriocettivo»; dobbiamo aggiungere che si tratta del sé che comporta «il senso delle possibilità motrici», così come delle «posture» e delle «facoltà» del corpo, e non ancora il senso delle «particolarità visive di qualcuno» (Gallagher e Meltzoff 2010: 117). Dagli studi riportati, basati anche su quelli precedenti di Meltzoff (1990) e Campbell (1995), consegue che la «prima nozione esclusivamente visiva di sé» è solo qualcosa di legato ad uno «stadio dello specchio tardivo» o ad una «forma più tardiva di imitazione»: «l'auto-riconoscimento nello

9 In una nota di lavoro (VI: 267, maggio 1960) de Il visibile e l'invisibile, il filosofo francese conferma sostanzialmente questo punto di vista. Sostiene che il «toccarsi», come «il vedersi» è ottenere la «carne», «fenomeno di specchio», e «lo specchio è estensione del mio rapporto con il mio corpo». Certamente, dice che la «fissione dell'apparenza e dell'Essere» accade «già nel tatto (dualità del toccante e del toccato)»; tuttavia, riafferma che «con lo specchio (Narciso)» vi è una «più profonda aderenza a Sé». (D'ora in avanti, quando citiamo le "Note di lavoro", alla sigla dell'opera seguirà la data). Ne L'Occhio e lo Spirito ribadisce ancora che la «visione è l'incontro di tutti gli aspetti dell'Essere, come a un crocevia» (OS: 59). 
specchio è solamente una misura, un aspetto di un concetto più largo del sé» (Gallagher e Meltzoff 2010: 117). Sul piano neuroscientifico, guardando all'ontogenesi del sistema nervoso centrale, con i dati ricordati precedentemente sembra confermarsi anche l'ipotesi secondo la quale la «connettività specifica» che può svilupparsi durante l'ultima fase della gestazione fra le «regioni motorie» e «quelle che diverranno visive» del cervello esprimerebbe l' «architettura funzionale» su cui si costruiscono successivamente, per l'appunto, «aree del cervello che, una volta raggiunte dagli stimoli visivi dopo la nascita, sarebbero pronte a rispondere specificamente all'osservazione di movimenti biologici come quelli delle mani o [del]le espressioni facciali» (Gallese 2009, p.191). ${ }^{10}$ Al riguardo, spiega il neuroscienziato in un'intervista: quando, immediatamente dopo la nascita, il sistema motorio del bambino «è stato attivato dall'osservazione di un gesto mai visto prima», esso "è processato come tale dal sistema visivo perché il sistema motorio gli ha insegnato a "riconoscerlo"» (Lavazza 2010). Ci imbattiamo in un' organizzazione funzionale motoria precoce

10 Richiamando i risultati di Zola (cfr. Zola et al. 2007), scriveva Gallese, co-autore con Castiello e altri colleghi della pubblicazione sulla formazione dell'azione sociale nel parto plurigemellare (cfr. Castiello et al, 2007): «Si potrebbe ipotizzare che durante lo sviluppo prenatale connessioni specifiche possono svilupparsi fra i centri motori che controllano la bocca, ed i comportamenti della mano diretti ad uno scopo e le regioni cerebrali che diverranno ricettive verso gli stimoli visivi dopo la nascita. Tale connettività potrebbe fornire l'architettura funzionale (per esempio profili spazio-temporali specifici di scariche neuronali) ad aree del cervello che, una volta raggiunte dagli stimoli visivi dopo la nascita, sarebbero pronte a rispondere specificamente all'osservazione di movimenti biologici come quelli delle mani o [del]le espressioni facciali. In altre parole, i neonati e gli infanti, attraverso una connettività specifica sviluppatasi durante l'ultima fase della gestazione fra le regioni motorie e "quelle che diverranno visive" del cervello, sarebbero pronti a rispecchiare e imitare i gesti eseguiti dagli adulti che se ne prendono cura, posti di fronte a loro, e sarebbero forniti delle risorse nervose che abilitano il comportamento reciproco che caratterizza la nostra vita postnatale fin dal suo primo inizio (Gallese 2009, p.191). 
che ci dice che l'altro da Sé fa sistema con noi ontogeneticamente a livello del sistema motorio, e non ancora di quello visuo-motorio.

Nel prenatale ritroviamo il vincolo del "Sensibile" recuperando finanche l'imbricazione carnale di corpo fenomenico e corpo fisico e oggettivo. In effetti, una volta condotta sul piano ontologico, è quest'imbricazione a riflettere la carne come «sé lacerato». La duplicità che si forma tra il "fenomenico" e 1"“oggettivo" nel prenatale non ci permette di scegliere tra l'essere senziente e l'essere sentito: la scelta tra il corpo completamente attivo e quello completamente passivo non ci restituisce il corpo (cfr. N: 323). Riteniamo che una tale impossibilità implichi che sia il corpo stesso, materiale e oggettivo, a trovare una riabilitazione nel filosofo francese, nella misura in cui 1' "animazione" della "cosa fisica" non avviene in opposizione alla carne: ${ }^{11}$ a questo livello di profondità esperienziale, lo schema corporeo testimonia un rapporto con il mondo come rapporto «con sé in generale» (ivi: 324), generalità d'essere. Se

11 Qui Merleau-Ponty raggiunge la maggiore distanza critica dalla filosofia che ha ripreso e sviluppato con il concetto di Leib, come ricorda la Depraz (2011: 386), la distinzione oppositiva Körper/Seele (corpo/alma). In questa prospettiva, contrariamente alla stessa posizione della Depraz (1995), i vari ambiti che interessano la passività devono farla ritornare alla radice più lunga dell' "istituzione" (Stiftung): si tratta dell'originario anonimo On da conciliare con la passività. Esso fa della soggettività corporea merleau-pontiana qualcosa di già incarnato, che genera l' "unità di senso" non ancora in virtù di una coscienza attualizzante, ma dell'anonimato "fondamentale" che passa tra la carne del mondo e la carne del corpo. Dell' "unità di senso" resta nel pensiero di Merleau-Ponty solo la fungenza, non l'intenzionalità (cfr. anche Saint Aubert 2013: in part. 157-158). Criticando Merleau-Ponty, la Depraz ha sostenuto invece che l'incarnazione non dovrebbe rientrare nell'ordine di «una costituzione passiva» pensata come «costituzione originariamente anonima», alla luce del fatto che Husserl non accetterebbe questa confusione di passività e anonimato (Depraz 1995: 275). Nella distinzione tra «costituzione passiva»e «costituzione anonima», l'incarnazione - seguendo Esperienza e Giudizio (cfr.Husserl 1995: §17) - è il processo per il quale, davanti all'essere che «mi dona delle sensazioni passive», la coscienza affetta dalle cose attualizza una di tali sensazioni «in vissuti di coscienza» (Depraz 1995: 275-276). 
l'«Essere verticale», pre-spirituale, è destinato a risolvere il tradizionale rapporto «fral'anima e il corpo» (VI: 246, gennaio 1960), correlativamente, nel rispetto della dialettica «a spirale $»^{12}$ tra l'inferiore e il superiore, l'anteriore e il successivo, in cui uno stadio sopraggiunge all'altro più che rimpiazzarlo, non vi sono semplici meccanismi corporei, ma imbricazione di organo e comportamento, ${ }^{13}$ come indicano gli studi di Arnold Gesell commentati anche da Raymond Ruyer (1953) e ripresi criticamente da Merleau-Ponty: «"la respirazione 'distratta"” non è mero «"funzionamento fisiologico"», ma è un "comportamento respiratorio», vive così di «"temi respiratori”» nei feti dei mammiferi (IP: 52, 130, n.18).

Correlativamente, nel prenatale possiamo rinvenire le versioni ultime della "promiscuità" merleau-pontiana, precedentemente evidenziate: sono quelle dell" "accoppiamento", di ciò che arriva ad essere pensato come "scambio sostanziale" del "mangiare l'altro", con Claude Simon e Paul Claudel, e della "pregnanza", la negatività operante, fungente (adesione per via di una mancanza intrinseca, costitutiva). Significativamente, Merleau-Ponty parla della «mescolanza» e del «magma degli uomini» riprendendo Claude Simon (PF: 200-203 passim), secondo - si sintetizza incisivamente - un «foglio spaziale o topologico, l'avvolgente-avvolto», e secondo un «foglio temporale o archeologico, il generante-generato»: «l'avvolgente-avvolto affiora dopo i suoi aspetti più arcaici, animali, addirittura vegetativi - la respirazione, il nutrimento -, e fin nell'accoppiamento». A sua volta, «il foglio più temporale del generante-generato culmina con la pregnanza», ma è già abbozzato dall'ambito dell'

12 Scrive Merleau-Ponty, commentando le ricerche di Gesell e Amatruda (1953): «Il comportamento si sviluppa "a spirale". Se è così, ogni tema motorio della vita embrionale può essere considerato come un tema che sarà elaborato a un livello superiore nella vita post-natale» (N. 218).

13 Nel commentare sempre le ricerche di Gesell e Amatruda (1953), Merleau-Ponty evidenzia l'imbricazione intraorganico-comportamentale: «lo sviluppo embrionale anticipa il comportamento futuro, gli organi o abbozzi di organi dell'embrione non hanno alcun senso se si considerano indipendentemente da tutta la logica del comportamento» (N: 217). 
«avvolgente-avvolto»: la respirazione, il nutrimento, l'accoppiamento comportano tante «reversibilità vitali (tra attivo e passivo, dedans e dehors, io e altri)» che finiscono per partecipare alla generazione di noi e di altri (Saint Aubert 2013: 370).

Ora, con il prenatale siamo sospinti a riconoscere le tante reversibilità vitali e l'iniziazione intraorganico-comportamentale, in cui non c'è possibilità di tracciare frontiere precise tra la maturazione dell'organismo e l'emergere del comportamento, nell'imbricazione del mantenersi in vita con il funzionare del corpo. Per Merleau-Ponty, allorquando ci imbattiamo nell' impossibilità di separare anatomia e funzione, finiamo per infrentare il problema della relazione tra lo stesso organismo preneurale e l'ambiente: vi è una «dinamica preneurale», alla luce della quale l'organizzazione del sistema nervoso non è «la spiegazione ultima», non essendo dovuta tanto al «funzionamento del neurone», dell' «eccitazione nervosa» in quanto tale, ma «alla crescita dell'organismo totale» ${ }^{14}(\mathrm{~N}$ : 211). In questa crescita, si deve recuperare la vita "passiva" e "anonima", nonché quel fondo naturale e inumano, di cui egli parlava sin dall'opera Fenomenologia della percezione. Ma se non possiamo scindere l'organico dal comportamentale, allora l'affermazione della vita fetale umana implica qualcosa che è "naturale" e "storico" allo stesso tempo. Specificamente, nel sorgere dal «c'è inaugurale» (VI: 251, gennaio 1960) e nell'essere ripresa da un portatore determinato, la vita trascina con sé la "passività originaria", in cui si annida l'imbricazione del "naturale" e dello "storico".

Se l'evoluzione degli organismi si intreccia con impulsi e motivazioni non solo attivati dal cervello, ma posti in relazione all'esplorazione dell'ambiente (cfr. Damasio 2011: 42-44), il quadro filosofico merleau-pontiano ci restituisce quest'intreccio rinvenibile nel prenatale alla luce della "praxis": con la coscienza dei propri limiti somatici, foriera della distinzione tra il suo Sé, l'ambiente intrauterino e l'altro Sé, il feto maturo

14 Merleau-Ponty fa riferimento alle ricerche di Coghill (1929). 
finisce per tracciare una "storia" sotto forma di eventi che condizioneranno altri eventi, sotto forma di appello ad un proseguimento, secondo la logica del "possibile". Non a caso, davanti alla difficoltà di porsi a livello dell'axolotl, Merleau-Ponty afferma che «la vita pone le basi della storia», anche se «la regolazione della specie non è onnipotente», trattandosi di «storia naturale», non di «storia dell'uomo», di «storia individuale». Inspessita così della logica del possibile, la vita percettiva stessa deve raccogliere la sfida rappresentata dalla giunzione di natura e storia fino al piano ontologico. Naturalmente, in questo quadro filosofico, il "campo", la "dimensione" non ci permettono di pensare in termini di pura attualità e presenza, ma solo in chiave di "Stiftung indiretta". Merleau-Ponty insegna a pensare l'incasso tra lo spazio e il tempo, essendo la loro relazione fatta di non attualità, di distanze, di rivalità, di "differenza" (l'ordine dell'incompossibile). Possiamo pensare lo spazio e il tempo solo come dimensioni che si incassano, secondo trasgressione (Überschreiten) e scarto (écart), perché anche lo spazio e il tempo devono rappresentare sempre una «sola grande differenziazione di un solo Essere» (PF: 189). La critica della pura attualità e presenza dischiude la logica del possibile.

Contro l'«ultimatum dell'ambiente all'organismo», il filosofo francese valorizza - richiamando ancora una volta le ricerche di George Ellett Coghill (1929) - il comportamento come «qualcosa che è in anticipo sul funzionamento, che comporta un riferimento al futuro, che è al di là dei possibili immediati e non può realizzare immediatamente tutto quello che tuttavia già abbozza». Se «c'è un'iniziativa, un carattere endogeno del comportamento», carattere che non lo riduce al «semplice effetto architettonico» né ad «un insieme di funzioni», ciò implica che l'organismo stesso, grazie alla sua «iniziativa endogena», potrà tracciare quella che «sarà la sua vita futura» (N: 223). Ora, il riferimento al futuro diventa il riferimento al possibile. A questo punto, è necessario passare per la nozione di istituzione.

Contro le «difficoltà della filosofia della coscienza» (LSN: 55), non superate in maniera soddisfacente nella Fenomenologia della percezione, 
la nozione di istituzione vuol ripensare «l'istituito» non come «il riflesso immediato» delle azioni specifiche del soggetto istituente, ma come ciò che «può essere ripreso in seguito da lui stesso o da altri senza che si tratti di una totale ricreazione». Ma togliere l'organismo dall'ordine naturale dell'immediato, significa anche restituirlo all'ordine storico della diacriticità: l'istituito «è quindi fra gli altri e me, fra me e me, come una cerniera, come la conseguenza e la garanzia della nostra appartenenza a uno stesso mondo». In tal maniera, la nozione di istituzione è tradotta da Merleau-Ponty con il termine Stiftung. Essa ci parla di una «fondazione» non solo di un sistema di reciproche inclusioni, tra l'appartenenza ad uno stesso mondo, ai diversi tempi e alle diverse temporalità, ma anche di «un ritmo di conservazione, ripresa e superamento degli eventi passati», che toglie, ad esempio, la pubertà dal piano puramente biologico (ivi: 55-56). Essa può esprimere così

gli eventi di un'esperienza che la dotano di dimensioni durevoli, in rapporto alle quali tutta una serie di altre esperienze avrà un senso, formerà un proseguimento pensabile o una storia - ovvero gli eventi che depositano in me un senso, non a titolo di sopravvivenza e di residuo, ma come appello a un proseguimento, come esigenza di un avvenire (ivi: 56).

Cosicché, nel prenatale, il feto deve mostrare come il possibile non possa essere pensato semplicemente nei termini di una ulteriorità dell'organismo, il che richiamerebbe indirettamente l'idea di un continuum positivo, pieno e determinato, ma deve seguire piuttosto la logica dell" "imminenza", quindi di una "transizione d'essere", in cui il corpo, nondimeno, è aperto a comportamenti possibili nell'esatta misura in cui questi ultimi vengono incorporati. Allora, la vita stessa fetale si riscopre, dal nostro punto di vista, come «apertura di un campo», «istituzione di un avvenire» (IP: 38).

Le dinamiche dell'istituzione, che Merleau-Ponty estende all'ordine animale ed umano, personale e pubblico, in cui l'esigenza o l'appello 
all'avvenire, alla cosa e all' Altro lasciano agire la totalità aperta, "dimensionale", trovano la loro radicalizzazione - nell' espressione del "trascendentale radicale" - sotto la forma del "fenomeno espressione". Produttore dell' «intimità di ogni espressione a ogni espressione», «appartenenza a un solo ordine» (S: 103), il "fenomeno espressione" trova la sua base epistemologica e ontologica proprio nell'enigma e nella moltiplicazione del sensibile. L'enigma sta nella "simultaneità" della nostra vita con gli altri e con il mondo, a partire dalla proprietà del corpo di essere la sede di sensazioni doppie, entrando in estesiologia con la cosa: percependo la cosa come sensibile aperta, il corpo fa sistema con essa. Significativamente, nei suoi primi Corsi al Collège de France, Merleau-Ponty mette in luce con la nozione d'espressione la necessità di restituire a certi elementi del percepito la «dignità di dimensione», per cui l'«adozione di un livello» suppone la «nostra installazione» nel percepito (MSME: 50).

Dal nostro punto di osservazione critico, si tratta di una transizione d'essere sensibile-senziente, senza un inizio assoluto, da riabilitare nella stessa processualità maturativa vitale del prenatale, in cui abbiamo ritrovato l'operato di "organizzatori” precoci dalle funzioni più varie e senza priorità (da quelle motorie a quelle oniriche). In luogo delle sole funzioni, vi sono relazioni d'essere (le relazioni sostenute dalla trascendenza dell'Essere) da esse veicolate, a vantaggio della segregazione "sensibile-senziente" e "senziente-sentito". A questo livello di profondità esperienziale ci pare che lavorino le intricazioni eminenti, ripensate filosoficamente, tra la vita percettiva, immaginaria e desiderante. In altri termini, se per Merleau-Ponty, la «vita percettiva» è ciò che agisce «al di qua e al di là dell'esercizio effettivo della sensorialità organica» (Saint Aubert 2013: 155), arrivando a pensare all' «onirismo» come «intricazione della vita immaginaria e della vita percettiva» (ibid.), ciò è vero proprio a livello del prenatale, che ci costringe a negare l'immagine-fantasma e a riconoscere che l'organo ha una funzione ontologica: il «carattere endogeno del comportamento» (N: 223), l'iniziativa che si produce tra l'organo e la funzione sono l'indice originale e originario 
di quella endo-percezione che si fa sul "vero dehors" e si scopre essere endo- proiezione, ciò che «gonfia l'essere percepito di una unità analoga a quella dello schema corporeo, e di un'interiorità che non è estranea alla nostra» (Saint Aubert 2011: 28). In definitiva, l'iniziazione intraorganicocomportamentale-ambientale si "istituisce" tra "natura" e "storia" per un sensibile che si fa senziente, emergendo dunque dal lato del dehors. Merleau-Ponty finisce per ritrovare 1'“apertura a", secondo profondità, dal lato del dehors, del «vero dehors» ${ }^{15}$, dunque della "profondità del mondo", in cui nasce la carne, nel rapporto di scarto con l'Essere a cui essa è rinviata ultimamente.

Se Mancia, per via del "Self neurobiologico", ritrova l'equilibrio che sostenta la vita per capire come il feto formi la coscienza dei propri limiti somatici (base della distinzione alla nascita tra il Sé e l'altro da Sé), e se Damasio vede, attraverso il "protoself", il recupero dell' "amministrazione" e della "preservazione" della vita in chiave di "valore biologico", di "principio", il quadro filosofico merleau-pontiano finisce per riabilitare queste "provocazioni" della scienza ${ }^{16}$, a patto però di obbligarci a ri-

15 Cfr. Etre et Monde (inedito, volume VI, B.N.F., cit. in Saint Aubert (2013: 377, n.1).

16 Risulta complesso il rapporto tra filosofia e scienza in Merleau-Ponty, che qui non abbiamo potuto prendere in considerazione. I dati espressivi sul prenatale, a partire dall'attribuzione al feto di una percezione di sé, distinta da quella dell'ambiente uterino e dell'altro da Sé, unitamente ad una azione orientata "socialmente", sono quelli di una scienza che provoca «la filosofia, la spinge a pensare i concetti validi nella situazione che è la sua» (N: 147); sono quelle di una "scienza che può fare soltanto "scoperte filosofiche negative"», che può dirci - parafrasando Merleau-Ponty - ciò che il prenatale non è, evitando di attribuirgli anche delle «evidenze» che mascherano delle «pseudo-evidenze», ma «non fornisce un'ontologia, neppure sotto forma negativa» (ivi: 156). Essendo espressione del "trascendentale radicale", la filosofia deve cercare il senso dei fenomeni descritti dalla scienza, ma nell'esatta misura in cui essa stessa è superata dal contatto con l'esperienza, sia vissuta che storica, perché, ultimamente, «l'originario esplode, e la filosofia deve accompagnare questa esplosione, questa non-coincidenza, questa differenziazione» (VI: 142). Scalzando la rivalità tra filosofia e scienza, assente comunque anche in Merleau-Ponty, il prenatale come 
pensare pienamente la logica della "nascita continuata" riconducendola all'Essere bruto, alla dimensionalità stessa bruta, selvaggia, non senza un problematico rapporto tra carne del mondo, del corpo e dello stesso Essere. A questo punto, va detto che il prenatale ci restituisce il fondo immemorabile e inumano, impercepito, senza dualismi e riduzionismi, cercato sin dalla Fenomenologia della percezione. Le possibili nozioni di inconscio e di memoria fetale ne sono la testimonianza.

Davanti all'importanza della «transmodalità sensoriale» studiata da Stern (1987), dell'integrazione di «varie esperienze sensoriali molto tempo prima dello sviluppo della visione», si sottolinea che proprio il ritardo di quest'ultima rispetto agli altri sensi fa sì che «il feto sia nella impossibilità di crearsi rappresentazioni visive». È ciò che permetterebbe di pensare le «"configurazioni neuronali" create dalle esperienze sensoriali», agenti come «base delle fantasie primarie (o protofantasie)». Si tratterebbe di «"fantasmi originari”» che affettano il feto, oggetto di certa psicoanalisi francese, e che inducono a pensare alla «memoria implicita», a quella memoria «il cui ricordo non potrà mai venire alla luce» (Mancia 2006: 214) ${ }^{17}$ Di qui, in prima battuta, la problematica merleau-pontiana dell'inconscio sarebbe comprensibile alla luce del fatto che la base dei primi contenuti sensoriali del feto, tra affetti e fantasie, è data ontogeneticamente dall' esperienza della costituzione dei «primi oggetti emozionali depositati nella sua memoria implicita, la prima a essere funzionante all'inizio della vita, per la precoce maturazione dell'amigdala» (cfr. Joseph 1996; cfr. LeDoux 1996; cfr. LeDoux 2000; cfr. Siegel 1999) ${ }^{18}$.

ambito di studio scientifico "provoca" la filosofia, inclusa quella merleau-pontiana, ma nell'esatta misura in cui bisogna pensare o ri-pensare filosoficamente (merleau-pontianamente) il senso che la scienza attribuisce al prenatale.

17 Mancia richiama il contributo di Bergeret (2004). Sui fantasmi originari del feto, rimandiamo a Bergeret e Houser (2004: 263-274).

18 Sono tutti autori richiamati da Mancia (2006: 214). La memoria implicita, che «non necessita delle strutture della memoria esplicita per strutturarsi», è indicata con «la maturazione della corteccia cerebrale a partire dalla $30^{\mathrm{a}}$ setti- 
Si tratta di una «memoria implicita di base» che rivaluta le emozioni: in essa si depositerebbero «le prime esperienze sensoriali e motorie del feto». Di conseguenza, essa "può costituire un primitivo abbozzo di una funzione inconscia non rimossa della mente, non passibile di ricordo» (Mancia 2006: 213). Tuttavia, in seconda battuta, dobbiamo notare che la figura merleau-pontiana della promiscuità problematizza finanche il peso dell'inconscio sul tempo.

L'ordine del vécu, nell'intreccio di motricità e percezione che si fa espressione ("relazione a") e nel correlato lungo cammino preparato dall'abitudine, fa del percepire un «non-sapere» (IP: 218); è il «"nonsapere" ${ }^{19}$ in cui la "promiscuità" è quella più estesa e, al contempo, più radicale: è la promiscuità dell'ignoranza con la familiarità (cfr. Saint Aubert 2013: 216), è la "promiscuità" che si incrocia con l'idea di un inconscio che interferisce nel funzionamento della memoria. La distinzione ultima è, notoriamente, quella tra due gradi di inconscio; distinzione che pretende radicalizzare la nozione di inconscio freudiana, permettendoci di evitare di trasformare finanche il non rimosso in dato positivo. Incisivamente, Merleau-Ponty, nella caratterizzazione ontologica dell'inconscio, riconduce quest'ultimo al «sentire stesso», ad un sentire che "non è il possesso intellettuale di "ciò che" è sentito, ma spossessamento di noi stessi a suo vantaggio, apertura a ciò che non abbiamo bisogno di pensare per riconoscere». E chiarisce che l' «inconscio di rimozione sarebbe quindi una formazione secondaria, contemporanea alla formazione di un sistema percezione-coscienza, e l'inconscio pri-

mana di gestazione, in particolare con la maturazione dell' amigdala che avviene molto tempo prima di quella dell'ippocampo». È una memoria che rimanda così all'amigdala, a un emisfero che presiede alle emozioni, interagendovi secondo connessioni reciproche. Inoltre, essa è collocata in «un circuito che, oltre all'amigdala, coinvolge i gangli della base, il cervelletto e aree corticali temporo - occipito - parietali dell'emisfero destro» (ivi: 212).

19 Al riguardo, Saint Aubert (2013: 216) cita dell'inedito Essere e Mondo una sequenza di lavoro di febbraio o marzo 1959. 
mordiale sarebbe il lasciar-essere, il sì iniziale, l'indivisione del sentire». Dunque, egli arriva a pensare che vi è una "dimensionalità" inconscia che scalza le «"rappresentazioni inconsce"», l'inconscio definito «in termini di "rappresentazioni inconsce", tributo pagato da Freud alla psicologia del suo tempo» (LSN: 129), così come scalza «un secondo "io penso" che saprebbe quanto noi ignoriamo di noi stessi» (ivi: 61), un «sapere», «un altro soggetto» e una «seconda coscienza» (IP: 215). Cosicché, la stessa idea di "proto-rappresentazione", legata alla coscienza a contenuto sensoriale del feto, viene meno. Tutto ciò è pensato a vantaggio dell'incarnazione dello schema corporeo, di una correlata «logica d'implicazione o di promiscuità» (LSN: 61). L'inconscio così inteso finisce per fondare nell'interrogazione merleau-pontiana la nostra appartenenza carnale, raffinata nel "transitivismo" secondo scarto o diacriticità: nel dover dar conto dell' «osmosi tra la vita anonima del corpo e la vita ufficiale della persona», l'inconscio freudiano è ciò che si è introdotto «tra l'organismo e noi stessi come sequenza di atti deliberati, di conoscenze espresse» (S: 300). In tal senso, esso s'impone come «l'eternità esistenziale, la coesione di una vita, la fecondità dell'evento» (IP: 223).

Di qui, se il sapere non riconosciuto lascia con sé un potere affetto dalla non passibilità del ricordo, allora esso risale alla produzione di quella "unità di senso" prenatale che avviene sul fondo della "processualità maturativa", vitale $e$ comportamentale, non di una memoria esplicita che la sostenga, né di un meccanismo di difesa. Ma affinché la funzione inconscia non rimossa della mente non ci restituisca un oblio come dato positivo, come l'oggetto (ob-jectum) o come "in sé", bisogna rimontare ai "temi" o a dei «nuclei di significazione», su cui si impernia «la vita trascendentale» stessa e in cui vi è l'iniziazione del «soggetto come campo, come sistema gerarchizzato di strutture aperte da un c'è inaugurale» (VI: 251, gennaio 1960). Allora questo sapere non riconosciuto né formulato può convergere sulla memoria implicita, a patto che non si intenda con essa la semplice "mancanza di", la mancanza di una memoria esplicita o l'annuncio di essa. In effetti, il problema della 
memoria deve scappare all'alternativa fra conservazione e costruzione: se si rinunciasse a pensare al presente in chiave di "rappresentazione» (Vorstellung), riallacciando i nostri rapporti spaziali e temporali con lo «schema posturale» (rifondato nella carne), «la vera memoria»-dice Merleau-Ponty - si ritroverebbe all' «intersezione» della «conservazione» e della «costruzione», «nell'istante in cui ritorna alla mente il ricordo dimenticato e conservato dall'oblio», perché «ricordo esplicito e oblio sono due modi della nostra relazione obliqua con un passato che non ci è presente se non per il vuoto determinato che lascia in noi» (LSN: 62 63). Se ciò permette di non trattare la memoria implicita come un inizio che non è mai esistito, allora vi è una "fungenza" realizzata dalla processualità vitale stessa sin dal non rimosso: la memoria implicita finisce per confermare che vi è una processualità maturativa vitale "operante", "fungente" nella misura in cui trascina con sé emblematicamente l'imbricazione originalmente e originariamente non rimossa dell'organico con il comportamentale, naturale $e$ storica, e con essa il vécu come "tema". Una tale memoria ci dice che bisogna procedere non solo oltre l'oblio come dato positivo, presenza incondizionata, ma primieramente oltre la riduzione dell'organismo stesso alla fisiologia pura e all'apprendimento puro, nonché all'ordine puntuale e localizzabile.

A questo punto, anche un possibile concetto di "fantasma originario" risulta superabile. Contro l'interpretazione della carne come nozione in cui ritrovare un «fantasma delle origini», dunque «l'origine di ogni fantasma» (Saint Aubert 2013: 339-340), ${ }^{20}$ Merleau-Ponty pensa all' «apertura al mondo» del corpo percipiente (percettiva, espressiva, desiderante) abitato sì dai rapporti originari con il corpo materno, ma in chiave strutturale («archeologia» e «topologia» materne), non di «"fusione"» e «"chiusura"». ${ }^{21}$ In sostanza, con la filosofia merleau-pontiana

20 È l'interpetazione che Pontalis (1971) ha dato della nozione di carne di Merleau-Ponty.

21 Di fusione e chiusura parla Sichère (1982), qui citato e criticato da Saint Aubert (2013: 340-341). 
siamo spinti a «ritrovare nell'immanenza della carne una trascendenza nascosta, nella sua circolarità uno scarto e un'apertura» (Saint Aubert 2013: 341). L'inconscio come sapere non riconosciuto, non formulato, che trascina con sé finanche il non rimosso a livello della memoria implicita, è saldato nella comunione vitale (fisiologica $e$ psicologica, percettiva e "praxica") in cui vive il feto con il corpo della madre attraverso le "funzioni di contenimento", nonché nella comunione originalmente e originariamente "sociale" (con la madre e l'altro o gli altri feti). ${ }^{22} \mathrm{Ci}$ limitiamo ad evidenziare che Merleau-Ponty spinge a pensare - congiuntamente all'interpretazione di Freud - al rovesciamento o chiasma tra il «per-Sé per-Altri», a vantaggio del «co-funzionamento», funzionamento di un «corpo unico» (VI: 229, $1^{\circ}$ novembre 1959). Tuttavia, se i dati empirici spingono a restituire l'aderenza carnale al feto (il "Sé"), le transizioni reversibili merleau-pontiane sussistenti nel mondo postnatale (tra l'esperienza passiva e quella attiva, tra il "didentro" e il "difuori" e tra il lato individuale e quello universale) risultano essere tardive, non solo sul piano cronologico, ma ultimanente sul piano trascendentale: abbiamo originalmente e originariamente la transizione continuamente reversibile dell'esperienza del feto verso l'ambiente vitale, che si fa ambiente di comportamento, e verso l'altro (il co-gemello o la madre) e indirettamente verso tutte le relazioni che la madre intrattiene con il mondo. In effetti, anche e soprattutto a questo livello non esiste il problema dell'alter ego: nell'intercorporeità, feto-madre, nell'unico corpo prenatale che si sdoppia, più che in un "corpo unico" formato dopo lo sdoppiamento postnatale, non è propriamente il feto o i feti che sentono, né la sola madre che sente, nella misura in cui sono abitati da un sentire "anonimo" e "generale". S'impone l'entropatia (Einfühlung) sin dal mondo prenatale.

22 Sono aspetti che qui non possiamo sviluppare sul piano scientifico, tantomeno sul piano filosofico dell'endo-ontologia merleau-pontiana. Parlando di carne del feto e di carne dell'utero-mondo, abbiamo tentato di pensare i primi possibili sviluppi ontologici del prenatale attraverso Merleau-Ponty in un saggio di recente pubblicazione (cfr. Di Clemente: 2016). 
Sul solco dell'insegnamento merleau-pontiano, nel prenatale l'entropatia verrebbe ricondotta esattamente alla co-nascita, e non ancora all'aspetto psicológico-affettivo; verrebbe ricondotta alle dinamiche interne alla comunione vitale comportamentale, foriera di una "intelligibilità allo stato nascente" e interna alla stessa processualità maturativa vitale di funzioni "antropologicamente" rilevanti (rilevanti come "fenomeno espressione"), da cui abbiamo visto emergere l'interazione sensomotoria materno-fetale. Naturalmente, essendo la processualità "fungente", "operante" a livello di transizioni d'essere, l'entropatia verrebbe radicata sempre nella reversibilità o metamorfosi nel tessuto unico o carnale del sentire, in cui vi è "sopravanzamento", "trasgressione intenzionale" tra il sentire dell'uno e dell'altro (cfr. ivi: 218, 219, due "Note di lavoro" di settembre 1959). Rinviata ad un tale terreno originale e originario, la reversibilità che passa nella comunione vitale stessa tra feto o feti e madre può costituire allora l'emergenza dell'entropatia. Quest'ultima può scoprirsi "radicalmente" come operazione "quase-corporea": è estesiologica prima ancora che psicológico-affettiva. Complessivamente, il feto è toccato, animato dall'utero-mondo per "analogia" 23 , non diversamente da ciò che Merleau-Ponty dice delle cose: «esse sono ciò su cui si apre la Sinergia del mio corpo, sono fatte della stessa stoffa dello schema corporeo, io le abito a distanza ed esse mi abitano a distanza». Dobbiamo recuperare finanche il rapporto di Einfühlung con l'utero -mondo, con le cose. Riferendosi agli animali, alle piante, oltre che all'altro, egli dichiara al riguardo: «il mio interno è l'eco del loro» (N: 325; cfr. anche PF: 60). Evidentemen-

23 Sul problema dell' analogia, Saint Aubert nota che ci troviamo di fronte, nell'ultimo Merleau-Ponty, al recupero della «vitalità immaginaria dell'analogicità, del "mondo onirico dell'analogia"», contro l'«uso sartriano dell'analogon», ovvero al recupero della sua "potenza metamorfica», persa nelle avventure dell'analogia entis. È una perdita di potenza che lo studioso ritrova soprattutto nei tentativi di affrontate le modalità umane della conoscenza di Dio senza che l'empiétement incida sull' «immutabile trascendenza di un Essere impassibile» (Saint Aubert 2013: 246) 
te, è l'Essere di promiscuità da ritrovare nel prenatale, quell'Essere di transitivismo, sensibile e originariamente verticale, che contiene duplici rapporti, secondo il «duplice chiasma» indicato da Merleau-Ponty per il solo mondo postnatale: non c'è solo lo «scambio me altro», ma «anche scambio fra me e mondo, fra il corpo fenomenico e il corpo "oggettivo", fra il percipiente e il percepito» (VI: 229, $1^{\text {o }}$ novembre 1959).

In sostanza, se la questione ruota intorno al comportamento che sconnette «certe comodità del pensiero» (N: 232), allora è la vita stessa come processualità maturativa di un portatore determinato (produttrice, emblematicamente, della giunzione di anatomia e funzione, organo e comportamento, e dell'identità tra il mantenersi in vita e il funzionare) ad aprire "dimensioni inedite", a vantaggio di una coscienza percettiva non regionale. Nell' iniziazione intraorganico-comportamentale bisogna ritrovare quel fondo fungente, figurale o gestaltista, in cui i vari aspetti del vegetativo, motorio, sensoriale, onirico, etc. dovrebbero essere riletti filosoficamente come un "fenomeno espressione" endogeno-ontologico: come l'intimità di un' espressione con le altre, iniziazione dell'essere percipiente ad un livello espressivo ("la relazione a"), sempre a vantaggio di una coscienza percettiva non più "regionale". Riteniamo che il "fenomeno espressione" endogeno-ontologico abbia il feto come portatore determinato di una negatività ontologica, perché il feto ha quel «significato ontologico», cercato dal filosofo francese, che lo rende irriducibile al «fatto empirico» (VI: 267, maggio 1960) e che fa sì che l'incorporazione si manifesti in veste di carne, nell'apprensione indiretta della "carne del mondo", di quell'utero-mondo da riqualificare come "senso in ciò che è esterno". Incisivamente, volendo limitarci a riprendere i termini del discorso merleau-pontiano, il rapporto «non gerarchico» che fa essere, come lasciar-essere, al di qua del sì e del no, si ritrova nel prenatale, prima nel senso «laterale» o dell' «Ineinander» (N: 391) e poi, ultimamente, nel senso "figurale" o "gestaltista". ${ }^{24}$

24 Sugli sviluppi dell'incorporeo o degli incorporei (l'“ombra", la "profondità"), 
Del resto, Merleau-Ponty non rinuncia a pensare nel continuum prenatale-postnatale, non positivo, 1'“unità di senso". Egli scrive:

Quando l'organismo dell'embrione si mette a percepire, non c'è creazione da parte del corpo in sé di un Per Sé, e non c'è discesa nel corpo di un'anima prestabilita, c'è che tutto a un tratto il vortice dell'embriogenesi si centra nella cavità interna che essa preparava Un certo scarto fondamentale, una certa dissonanza costitutiva emerge (VI: 246-247, gennaio 1960).

In conclusione, nonostante le riserve sulla fase in cui si sviluppa la differenziazione tra il Sé, l'ambiente di comportamento e l'altro da Sé, il prenatale può mostrare agli occhi di Merleau-Ponty che il legame tra l'anima e il corpo non è quello tra due ordini, «ciascuno dei quali sarebbe autosufficiente», ma quello interno al "sensibile che si scava": è il legame preso in un "campo", in una "dimensione" correlabile al postnatale, in cui vi è il «legame del convesso e del concavo, della volta solida e della cavità che essa forma» ( ibid.), arrivando fino alla formazione "figurale" o "gestaltista" dell'Essere. A questo livello di originalità e originarietà esperienziale, primaria, andrebbe ricollocata finanche una tale scaturigine "figurale", "incorporea" dell'Essere, fondando così in chiave trascendentale per "analogia" (per trasferimento o generalizzazione) del Sé prenatale i possibili percorsi del voyant-vu postnatale. Al contempo, davanti al privilegio del "Sé" postnatale, dell'“aderenza carnale" postnatale, tutto ciò rimane solo un'anticipazione e non una "fondazione originaria" nell' interrogazione merleau-pontiana.

\section{Referenze bibliografiche}

BERGERET, J. Les "souvenirs/non - souvenirs". Colloque du Vinatier. Lyon, 27 novembre, 2004.

come ultimi aspetti dell'Essere, a servizio di un «modello figurale» o «gestaltista» dell'essere, rimandiamo a Saint Aubert (2013: in part. 398-408). 
BERGERET, J., HOUSER M. 2004. Le Fœtus dans nostre Inconscient. Paris: Dunod, 2004.

BURGESS, J., TAWIA, S.A. «When Did You First Begin to Feel It? Locating The Beginning of Human Consciousness». Bioethics. Vol. 10. Oxford: Blackwell Publishers, 1996, pp. 1-26.

BUSNEL, M-C. "A sensorialidade do Feto e do Recém-Nascido". In: Busnel, M.C. Yasaku, S., \& Cunha, I. Relação Mae-Feto. Visão atual das neurociências. (L'edizione accoglie anche: Humanizaçao do Nascimento). 2 ed. São Paulo. Casa do Psicólogo, 2008, pp. 13-30.

CAMPBELL, J. «The body image and self-consciousness». In: Bermúdez J., Marcel A. \& Eilan N. The Body and the Self. Cambridge (Mass.): MIT-Bradford Press, 1995, pp. 29-42.

CASTIELLO, U. et al. «Wired to Be Social: The Ontogeny of Human Interaction». PLoS ONE, 5-10 (2010): e13199.

COGHILL, G.E. Anatomy and the Problem of Behaviour. London: Macmillan, 1929.

DAMASIO, A.R. E o cérebro criou o homem. Trad. port. di L. Teixeira Motta. São Paulo: Companhia das Letras, 2011.

DE CASPER, A.J., FIFER, W.P. «Of Human Bonding: Newborns Prefer Their Mothers'Voices». Science, 208, 1980, pp. 1.174-1.176.

DEPRAZ, N. Transcendance et incarnation. Le statut de l'intersubjectivité comme altérité à soi chez Husserl. Paris: Vrin, 1995.

. «Postface: La traduction de Leib, une Crux phaenomenologica». In: E. Husserl. Sur l'intersubjectivité. Tome I. Traduction, Introduction, Postface et Index par N. Depraz. 2a ed. Paris: P.U.F., 2011, pp. 385-399.

DE VRIES, J.I.P., VISSER, G.H.A., PRECHTL, H.F.R. «The Emergence of Fetal Behavior». II. Quantitative Aspects. Early Human Development, 12, 1985, pp. 99-120. 
DI CLEMENTE, F. «La carne del feto. Riflessioni sul prenatale attraverso Merleau-Ponty». Sofia, vol. 6, n. 1, Jan. - Jul., 2016, pp. 211-237. GALLAGHER, S., ZAHAVI, D. La mente fenomenologica. Filosofia della mente e scienze cognitive. Trad. it. di P. Pedrini. Milano: Raffaello Cortina Editore, 2009.

GALLAGHER, S., MELTZOFF, A. «Le sens précoce de soi et d'autrui. Merleau-Ponty et les études développementales récentes». In: B. Andrieu (textes réunis par). Philosophie du corps. Paris: Vrin, 2010, pp. 83-127.

GALLESE, V. I neuroni specchio e l'ipotesi dello sfruttamento neurale: dalla simulazione incarnata alla cognizione sociale. Trad. it. di M. Salucci. Acesso in 18/07/2016. Il presente contributo è stato pubblicato nella versione originale in: J. Pineda (a cura di). Mirror Neuron Systems: The Role of Mirroring processes in Social Cognition. Totowa NY: The Humana Press, 2009.

GESELL, A., AMATRUDA, C.S. Embryologie du comportement. Trad. fr. di P. Chauchard. Paris: P.U.F, 1953.

HERBINET, E., BUSNEL, M-C. (a cura di). L'alba dei sensi. Opera collettiva sulle percezioni sensoriali fetali e neonatali. Versione italiana curata da C. Bellieni. Trad. it. di S. Moretti. Siena: Cantagalli, 2001.

HUSSERL, E. Esperienza e giudizio. Ricerche sulla genealogia della logica redatte ed edite da L. Landgrebe. Trad. it. F. Costa, L. Samonà. Milano: Studi Bompiani, 1995.

. Meditazioni cartesiane. Nuova edizione it. a cura di F. Costa. Presentazione di R. Cristin. $3^{a}$ ed. riveduta. Milano: Studi Bompiani, 1997.

Idee per una fenomenologia pura e per una filosofia fenomenologica. Libro terzo: La fenomenologia e i fondamenti delle scienze. Vol. 2. Nuova edizione it. a cura di V. Costa. Torino: Einaudi, 2002. 
HUSSERL, E. E. Sur l'intersubjectivité. Tome I. Traduction, Introduction, Postface et Index par N. Depraz. $2^{\mathrm{a}}$ ed. Paris: P.U.F., 2011.

IANNIRUBERTO, A., TAJANI, E. «Ultrasonographic Study of Fetal Movements». Seminars in Perinatology, 5, 1981, pp. 175 - 181.

JOSEPH, R. «The Brainstem, Midbrain, Da, 5ht, Ne, Cranial Nerves, and Cerebellum». In: Joseph R (ed.). Neuropsychiatry, Neuropsychology, Clinical Neuroscience. Baltimore (MD): William \& Wilkins, 1996, pp. 354-392.

. «Fetal Brain Behavior and Cognitive Development». Developmental Review, 20, 2000, pp. 81-98.

KOUPERNICK, C., ARFOUILLOUX, J.C. «Neurobiologie et neurologie du développement». In: Gratiot-Alphandery, H., Zazzo R. (eds). Traité de Psychologie de l'Enfant. Vol. 2. Paris: P.U.F., 1970.

LAVAZZA, A. "Così i gemelli fanno amicizia già nel grembo". Intervista a V.Gallese e C. Becchio. Regina Mundi. La Rassegna stampa Cattolica per la Società, Attualità e Famiglia, 2010. (Tratto da: L'Avvenire del 14/04/2010). Accesso in 6/06/2016.

LEDOUX, J.E. The Emotional Brain. New York: Simon and Schuster, 1996.

. «Emotion Circuits in the Brain», Annu. Rev. Neurosci., 23, 2000, pp. $155-184$.

MANCIA, M. «Funzioni integrative del cervello e origine dello psichismo fetale: riflessioni teoriche e cliniche». In: G. B. La Sala et al. (a cura di). La «normale» complessità del venire al mondo. Incontro tra scienze mediche e scienze umane. Milano: Guerini Studio, 2006, pp. 207-220.

MANFREDI, P., IMBASCIATI, A. Il feto ci ascolta ...e impara. Genitorialità, transgenerazionalità e ricerca sperimentale. Roma: Borla, 2012. 
MELTZOFF, A. «Foundations for developing a concept of self: The role of imitation in relating self to other and the value of social mirroring, social modeling, and self practice in infancy». In: D. Cicchetti, M. Beeghly (eds). The Self in Transition: Infancy to Childhood. Chicago: University of Chicago Press, 1990, pp.139-164.

MERLEAU-PONTY, M. La prose du monde. Texte établi et présenté par Claude Lefort. Paris: Gallimard, 1969 [PM].

.M. Merleau-Ponty à la Sorbonne, résumé de cours (19491952), Grenoble: Cynara, 1988 [RCS].

[OS].

. L'Occhio e lo Spirito. Trad. it. di A. Sordini. Milano: SE, 1989

. Linguaggio, Storia, Natura. Corsi al Collège de France, 1952 - 1961. Traduzione, presentazione e note a cura di M. Carbone. Milano: Studi Bompiani, 1995 [LSN].

. La natura. Lezioni al Collège de France 1956-1960. Testo stabilito e annotato da D. Séglard. Ed. it. a cura di M. Carbone. Trad. it. di M. Mazzocut-Mis e F. Sossi. Milano: Raffaello Cortina Editore, 1996 [N].

. Les relations avec autrui chez l'enfant. Centre de Documentation Universitaire, 1951-1962. In: Parcours 1935-1951. Lagrasse: Verdier, 1997 [RAE].

. Il visibile e l'invisibile. Trad. it. riveduta da M. Carbone. $2^{\mathrm{a}}$ ed. Milano: Studi Bompiani, 1999 [VI].

. L'union de l'âme et du corps chez Malebranche, Biran et Bergson. Notes prises au cours de M. Merleau-Ponty à l'École Normale Supérieure $(1947-48)$. Notes recueilles et rédigées par J. Deprun. $2^{\mathrm{a}}$ ed. Paris: J. Vrin, 2002 [UAC].

MERLEAU-PONTY, M. Fenomenologia della percezione. Trad. it. di A. Bonomi. Milano: Studi Bompiani, 2003 [FP]. 
. Segni. A cura di A. Bonomi. Trad. it. di G. Alfieri. $2^{\text {a }}$ ed. Milano: il Saggiatore, 2003 [S].

. È possibile oggi la filosofia? Lezioni al Collège de France, 1958 - 1959 e 1960 - 1961. Edizione italiana e Introduzione a cura di M. Carbone. Prefazione di C. Lefort. Trad. it. di F. Paracchini e A. Pinotti. Milano: Raffaello Cortina Editore, 2003 [PF].

. L'institution. La passivité. Notes de cours au Collège de France (1954-1955). Textes établis par Dominique Darmaillacq, Claude Lefort et Stéphanie Ménasé. Préface de Claude Lefort. Paris: Belin, 2003 [IP].

. La struttura del comportamento. Trad. it. di G. Neri rivista da M. Ghilardi. Edizione a cura di M. Ghilardi e L. Taddio. Milano-Udine: Mimesis Edizioni, 2010 [SC].

. Le monde sensible et le monde de l'expression. Cours au College de France. Notes, 1953. Texte établi et annoté par Emmanuel de Saint Aubert et Stefan Kristensen. Avant-propos d'Emmanuel de Saint Aubert. Genève: MētisPresses, 2011 [MSME].

MILANI COMPARETTI, A. «Interpretazione funzionale dei movimenti fetali». Età Evolutiva, Convegno, n 10, 1981, pp. 88-92.

MONTAGU, A. Touching: The Human Significance of the Skin. New York: Harper and Row, 1978.

PIONTELLI, A. «Sull'inizio del comportamento fetale umano». In: M. Mancia (a cura di). Psicoanalisi e Neuroscienze. Traduzione a cura di Mariella Schepisi. Milano: Springer, 2007, pp. 413-442.

PONTALIS, J. B. «Présence, entre les signes, absence». L'Arc, n46, 1971, pp.56-66.

RIGHETTI, P. L. Elementi di psicologia prenatale. Roma: Edizioni Scientifiche Magi, 2003.

RUYER, R. «Les conceptions nouvelles de l'instinct». Les Temps modernes, n 96, nov. 1953. 
SAINT AUBERT, E.DE. “Avant-propos”. In: Le monde sensible et le monde de l'expression. Cours au College de France. Notes, 1953. Texte établi et annoté par Emmanuel de Saint Aubert et Stefan Kristensen. Avant-propos d'Emmanuel de Saint Aubert. Genève: MētisPresses, 2011, pp. 7-38.

. Être et chair I. Du corps au desir: l'habilitation ontologique de la chair. Paris: Vrin, 2013.

SCHAAL, B., HERTLING, E. "Nuovo-nato, nuovo naso? Olfatto e sviluppo precoce". In: Herbinet E. e Busnel M-C. (a cura di). L'alba dei sensi. Opera collettiva sulle ercezioni sensoriali fetali e neonatali. Versione italiana curata da C. Bellieni. Trad. it. di S. Moretti. Siena: Cantagalli, 2001.

SCHILDER, P. Immagine di sé e schema corporeo. Trad. it. di R. Cremante e M. Poli. Milano: Franco Angeli Editore, 1973.

SICHÈRE, B. Merleau-Ponty ou le corps de la philosophie. Paris: Grasset, 1982.

SIEGEL, S.J. The Developing Mind: Toward A Neurobiology of Interpersonal Experience. New York: Guilford, 1999.

STERN, D. Il mondo interpersonale del bambino. Torino: Bollati Boringhieri, 1987.

WALLON, H. Les origines du caractère chez l'enfant. Les préludes du

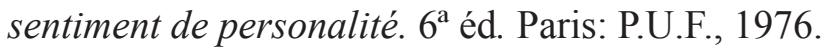

ZOIA, S. et al. Evidence of early development of action planning in the human foetus: a kinematic study, "Exp. Brain Res.”, 2007, 176, pp. 217-226.

Data de registro: 18/03/2015

Data de aceite: $20 / 11 / 2015$ 